\title{
A FLEXIBLE PROCEDURE FOR MULTIPLE CADAVERIC
}

\section{ORGAN PROCUREMENT}

\author{
Thomas E. Starzl, M.D., PH.D., F.A.C.S., Thomas R. Hakala, M.D., F.A.C.S., \\ Byers W. Shaw, Jr., M.D., Robert L. Hardesty, M.D., F.A.C.S., Thomas J. Rosenthal, M.D., \\ Bartley P. Griffith, M.D., Shunzaburo Iwatsuki, M.D., and \\ Henry T. Bahnson, M.D., F.A.C.s., Pittsburgh, Pennsylvania
}

Wirt the improvements in immunosuppression that have occurred in the last few years, a great increase can be expected in the demand for cadaveric kidneys and other vital organs. Doctor Koop, Surgeon General of the United States, has convened conferences and symposia to examine questions of cadaveric organ solicitation, removal, preservation and deployment. In such discussions, it has been obvious that a uniform procedure should be developed which is flexible enough to allow the excision of various organ combinations without jeopardy to any of the individual grafts. This study was done to report the method which we have used with satisfaction for several years.

\section{THE APPROPRIATE DONOR}

In the United States, it has become common to remove organs from so-called heart-beating cadavers with pronounced brain death. With some leisure, studies can be obtained in such donors of the function of individual organs. Cardiovascular-respiratory instability may be reflected in the necessity for vasopressor support, poor blood gas values or other adverse findings familiar to intensive care physicians. If such donors are unstable, kidney removal may be all that is possible. However, most brain dead donors can be maintained and improved with conventional intensive therapy and then an attempt can be made to co-ordinate the needs of surgeons who perform transplantations in different parts of the country in light of the availability of potential extrarenal grafts.

From the Department of Surgery, University Health Center of Pittsburgh, University of Pittsburgh, Pittsburgh.

This study was supported by research grants from the Veterans Administration and by Grant No. AM29961 from the National Institutes of Health, Bethesda, Maryland.

Reprint requests: Dr. Thomas E. Starzl, Department of Surgery, 3601 Fifth Avenue, Room 103, Falk Clinic, Pittsburgh, Pennsylvania 15213.
THE PRINCIPLE OF CORE COOLING

The intraoperative infusion of cold fluids is common to all preservation techniques. The most important objective is the avoidance of warm ischemia. Cooling of an organ graft by intravascular infusion of chilled lactated Ringer's solution at the time of circulatory arrest was introduced into the laboratory for experimental transplantation of the liver almost a quarter of a century ago (1) and was promptly applied clinically for the preservation of kidneys (2) and all other organs. Such cooling expands many fold the duration of organ viability and allows the unhurried application, if desired, of other more sophisticated preservation measures.

Lactated Ringer's solution has a low potassium content and is nearly isotonic. Chilled special solutions with an electrolyte composition similar to that in cells were shown in a study done in 1969 (3) to extend the permissable limit of cold renal ischemia beyond that achievable with isotonic solutions. The same effect has been shown with livers (4). Cardiac surgeons have cooled the heart with various cardioplegic solutions that have potassium concentrations of about 20 milliequivalents per liter.

The simplest and probably the best way to achieve immediate internal cooling of any organ is by in situ infusion with lactated Ringer's solution. Then, the individual organs may be infused with specified amounts of special solutions after their removal. The transplantation surgeons in the northeastern part of the United States prefer this approach since it avoids the uncontrolled infusion of large amounts of the potassium-rich Collins type solutions. However, the use of Collins solution for the in situ infusion of the donor procedure is so widespread that re-education away from this practice has been difficult. Furthermore, the establishment of a uniform code for infusion is not obligatory since the various solu- 


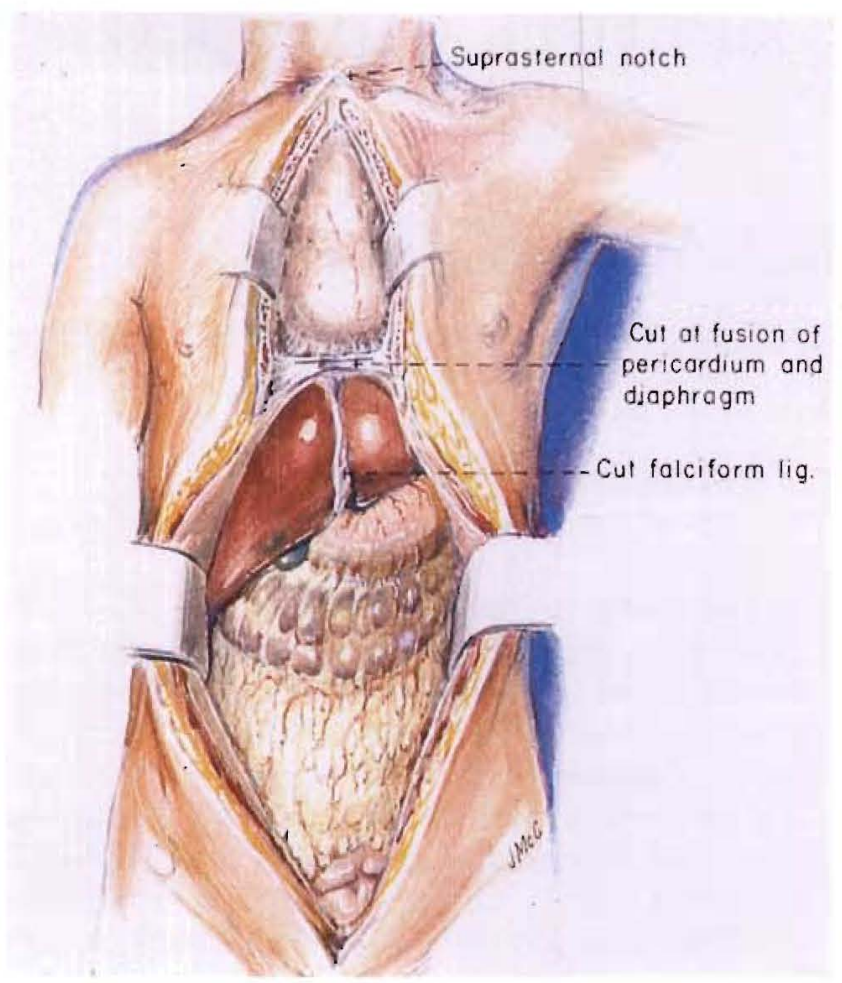

FIG. 1. Total midline incision used for multiple organ procurement.

tions can be used so easily for the in situ cooling of different organs in the same donor as will be made clear herein.

\section{THE INCISION}

A complete midline incision is made from the suprasternal notch to just above the symphysis pubis (Fig. 1). The sternum splitting component of the incision has replaced the cruciate abdominal incision which was commonly used previously. Most hospitals have a bone cutting saw for the sternum, but the Pittsburgh procurement team always carries a Lebsche knife and mallet plus a sternum spreader which have been useful when visiting small hospitals that do not have cardiothoracic equipment. The long incision provides good exposure for removal of the heart, both kidneys, the liver and other thoracoabdominal viscera.

\section{GRAFT NEPHRECTOMY}

All of the multiple organ removals can be envisioned as modifications of the evisceration techniques for cadaver kidney removal which have been described in other reports $(5,6)$.

The extraperitoneal space is entered by incising the peritoneal reflection of the ascending colon, cecum and distal part of the small intestine

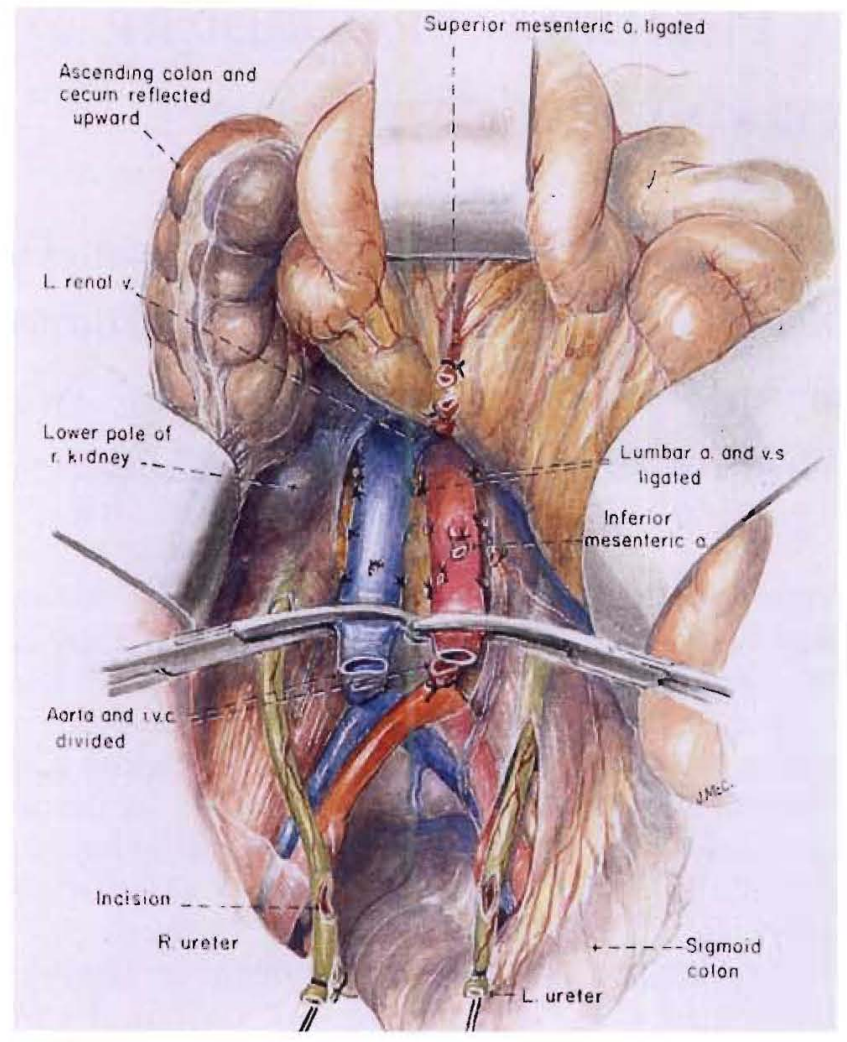

FIG. 2. Nephrectomy: preliminary steps for in situ infusion of kidneys in a cadaveric donor with an effective circulation who has been pronounced dead as a result of neurologic criteria.

(Fig. 2). The small and large intestine are swept up and the right ureter is identified, dissected distally and cut across near the bladder (Fig. 2). Its tip is tied with a long silk suture, and above this, an incision is made so that urine can escape. A urine culture may be obtained. The left ureter is treated in the same way either now (Fig. 2) or, if this is inconvenient because of the intact inferior mesenteric vessels, at a later time.

The distal part of the aorta and inferior vena cava are dissected free, encircled and divided (Fig. $2)$. Both great vessels are mobilized superiorly, ligating and dividing the inferior mesenteric artery and two or three sets of lumbar branches (Fig. 2). Upward dissection of the anterior surface of the aorta is continued until the left renal vein is encountered crossing the aorta from left to right (Fig. 2). Just superior to this, the superior mesenteric artery can be felt as a taut strand passing almost directly anteriorly providing the intestines are retracted anteriorly and superiorly. The superior mesenteric artery is encircled and ligated (Fig. 2). The viscera are returned to their natural location and attention is directed to a higher level.

The aorta is encircled either just above or just 


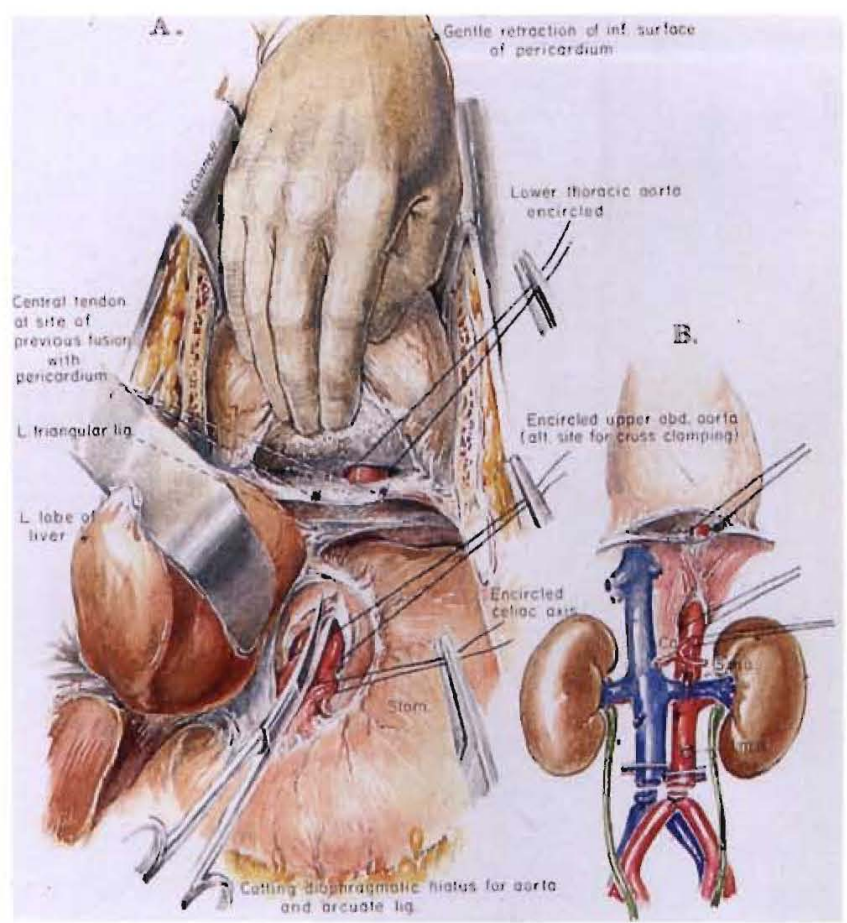

Fig. 3. A and B, Nephrectomy: preparation for in situ infusion by encircling the aorta just above or alternatively just below the diaphragm.

below the diaphragm. The easiest way to have perfect access to the aorta at the more superior level is to deepen the midline incision where the central tendon of the diaphragm fuses with the inferior surface of the pericardium. The fusion is opened up by separating the left part of the tendinous diaphragm from the pericardium, avoiding entry into the pericardial cavity if possible (Fig. 3). As the dissection proceeds leftward, the left side of the thoracic cavity is entered. The lower thoracic aorta is encountered immediately and encircled (Fig. 3).

Alternatively, the left triangular ligament of the liver may be incised and the upper part of the abdominal aorta can be encircled just above the origin of the celiac axis (Fig. 3A). This can be done safely after cutting the arcuate ligament and aortic hiatus (Fig. 3). When only the kidneys are to be removed, the celiac axis also should be encircled (Fig. 3A), ligated and divided.

The patient is now given 3 milligrams per kilogram of heparin. Cannulas are placed in the distal part of the aorta and the inferior vena cava (Fig. 4). The clamped aortic cannula is attached to an airfree infusion system through which chilled preservation solutions can later be infused (Fig. 4). The clamped vena caval cannula is attached to tubing that leads to a bleeding bag on the floor. An alpha-blocking agent can be given at

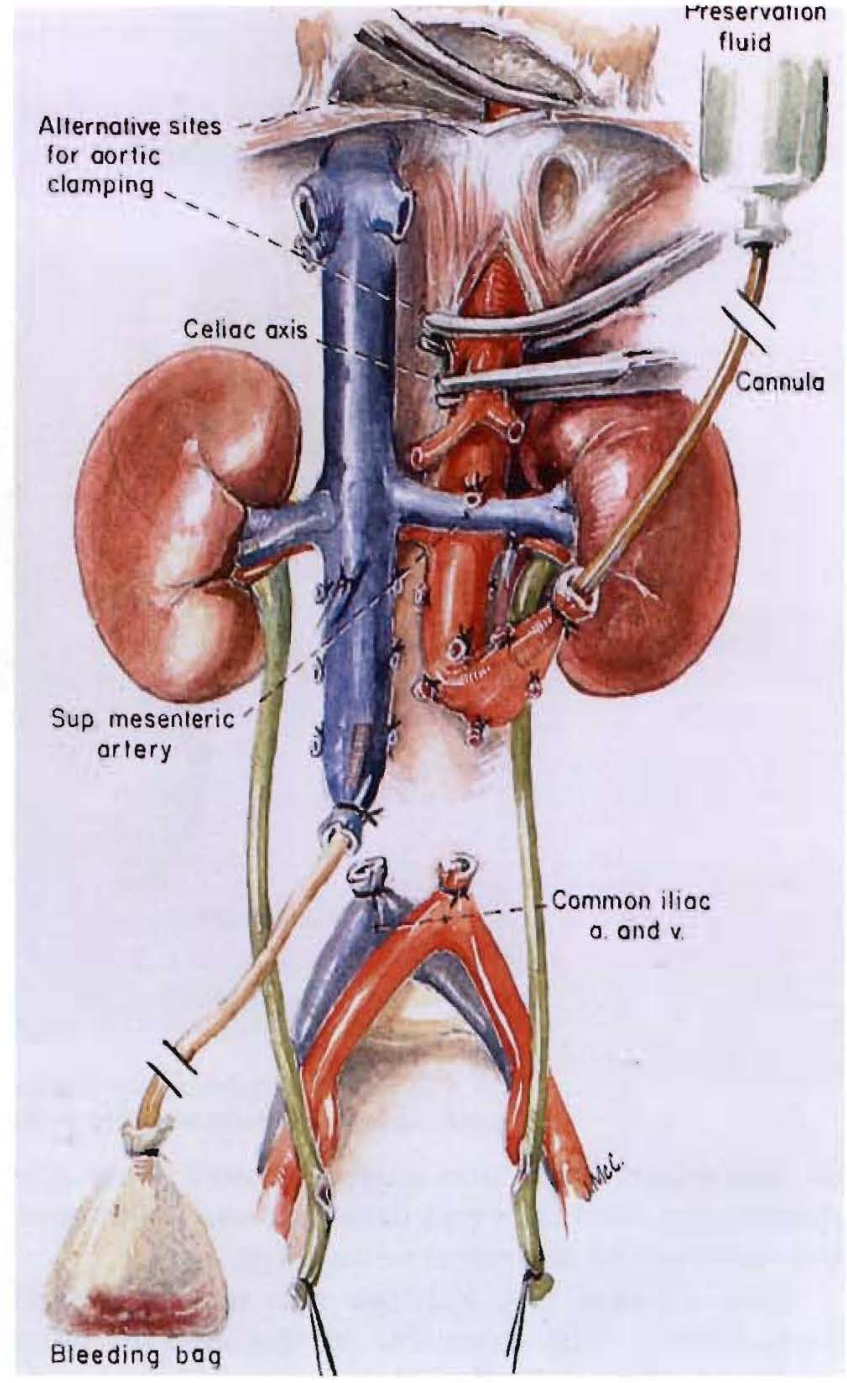

FIg. 4. Nephrectomy: in situ infusion of kidneys with cold preservation fluid through the distal part of the aorta, with a venous bleed-off from the distal inferior vena cava. The aorta is clamped proximally at one of the sites shown in Figure 3.

this time, and it is a common practice to give drugs, such as chlorpromazine, which are said to stabilize microsomal membranes.

If the kidneys are to be removed, no further dissection is necessary or desirable since infusion of the chilled preservation fluid will be done in situ. Either lactated Ringer's solution or one of the potassium-rich Collins solutions may be used. The aorta is cross clamped just above or below the diaphragm after making sure that the celiac axis and the superior mesenteric artery are ligated or clamped (Fig. 4). The chilled electrolyte solution passes predominantly into the renal arterial supply. The effluent is drained out through the inferior vena caval cannula to the bag on the floor. When in situ infusion of a liter or more in adults has been completed, the aorta is transected at one 


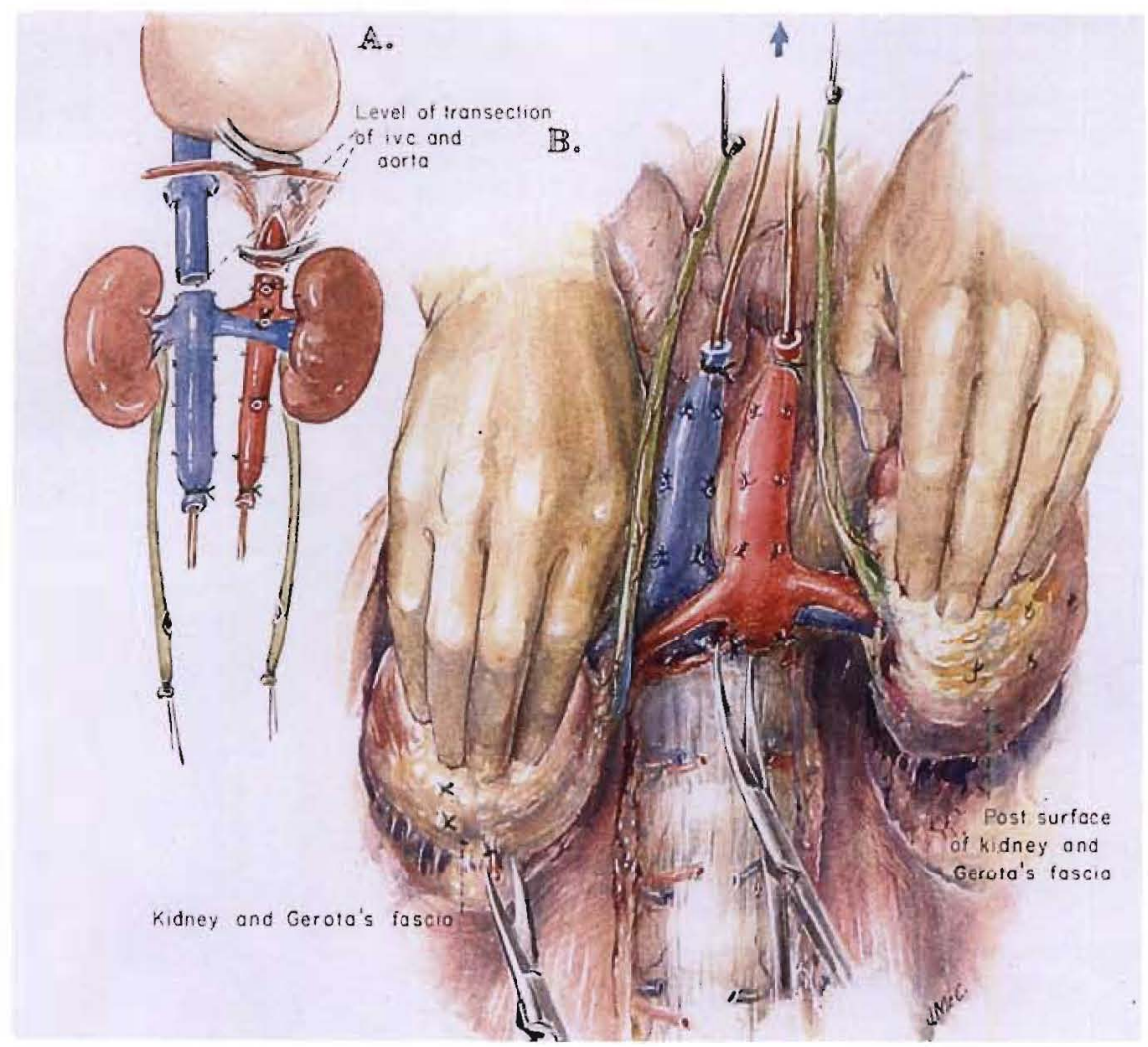

FIG. 5. A and B, Nephrectomy: removal of perfused kidneys en bloc. The kidneys and great vessels are held anterior to the plane of scissors dissection.

or the other level previously defined near the diaphragm, and the vena cava is transected above the entrance of the renal veins (Fig. 5A).

The washed out kidneys are now removed from below. The cannulas in the inferior vena cava and aorta as well as the ureters marked with ligatures are gently retracted anteriorly by an assistant who is asked to keep track of these four structures (Fig. 5B). In the meanwhile, the surgeon retracts one of the kidneys anteriorly with a hand placed behind Gerota's fascia and the nurse or another assistant performs the same task on the

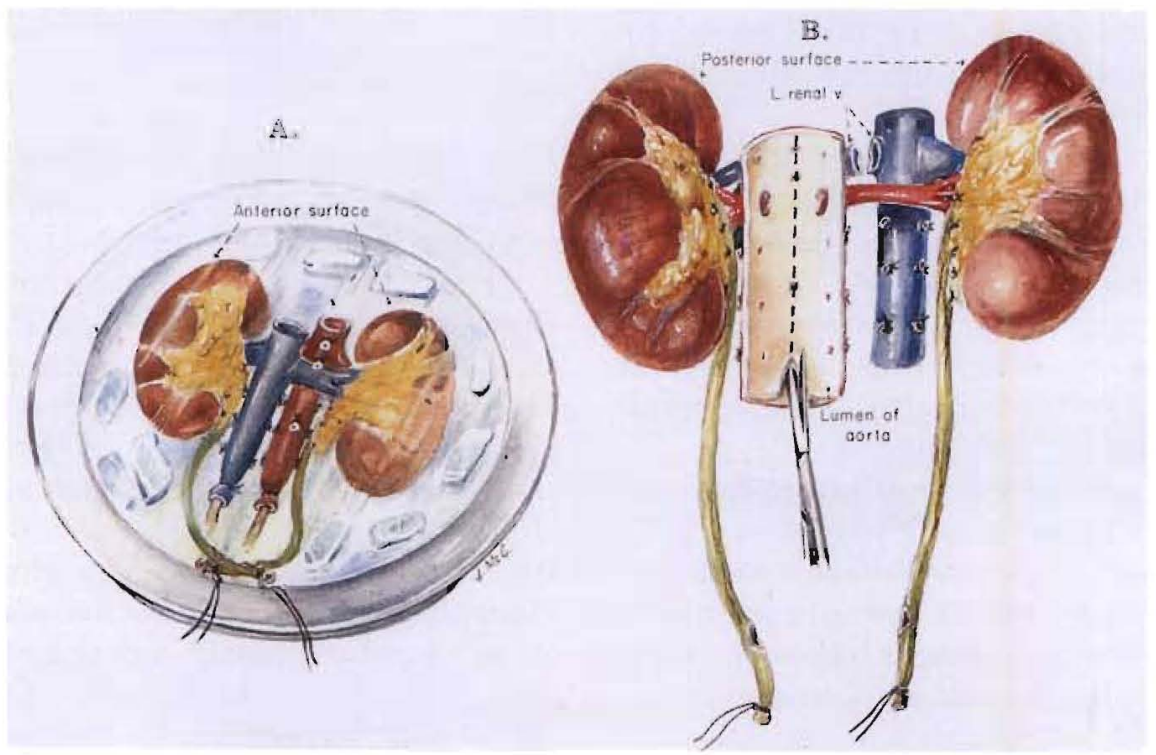

FiG. 6. A, Placement of kidneys in an ice basin. B, Division of kidneys using a posterior approach to split the aorta. 
other side (Fig. 5B). Neither the kidneys nor the four retracted structures are allowed to fall posteriorly at any time. All tissues passing posteriorly are cut, staying close to the ligaments and muscles covering the vertebral bodies (Fig. 5B) and continuing superiorly until the previously transected aorta and inferior vena cava are reached. Once the posterior dissection is completed, the flimsy residual tissues between the gastrointestinal tract and the anterior surface of the specimen are disconnected. The kidneys are taken from the body en bloc and placed in an ice basin on a back table (Fig. 6A). They can be prepared for machine perfusion of the entire specimen if this technique is preferred or the kidneys can be separated for either perfusion of the individual kidneys or preservation in slush. If the kidneys are to be separated, the left renal vein is transected flush at its entrance into the inferior vena cava (Fig. 6B). If slush preservation is planned for kidneys that were cooled by initial in situ infusion with lactated Ringer's solution, a final flush with one of the Collins type solutions is necessary.

For division of the kidneys for slush preservation, it is safest and most convenient to turn the specimen over (Fig. 6B). With one blade of a scissors inserted into its lumen, the posterior wall of the aorta is incised. A perfect guide to the line of aortic incision is the row of ligated or clipped

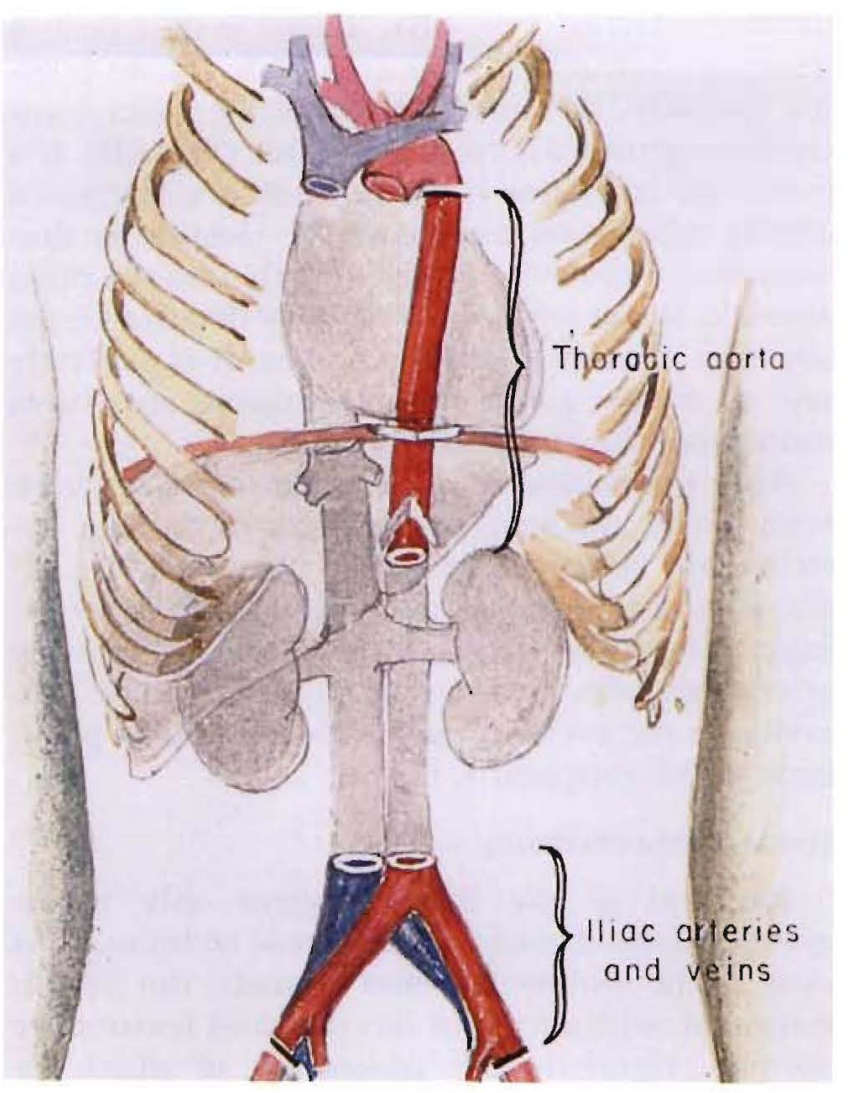

FIG. 7. Removal of segments of iliac arteries and veins and thoracic aorta. The vascular grafts are refrigerated and kept in case of an emergency.

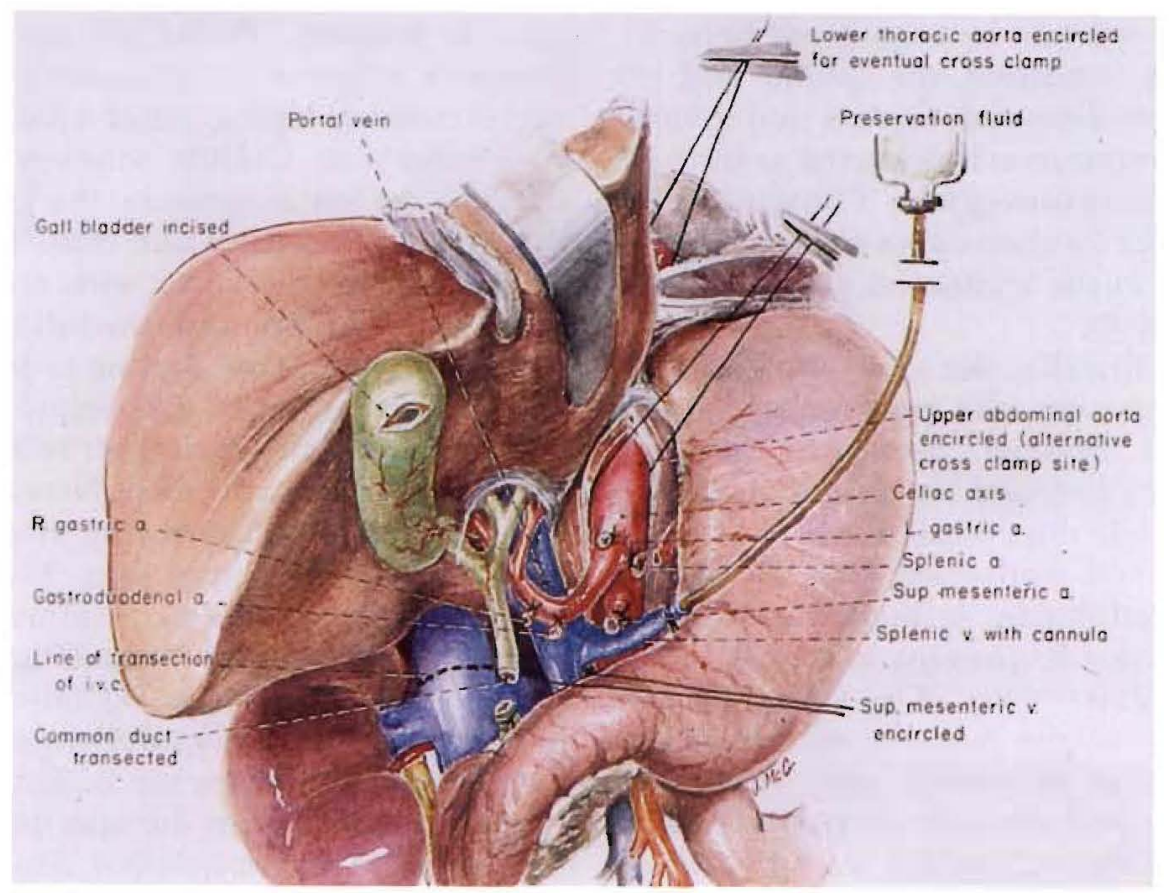

FIG. 8. Hepatectomy: hilar dissection and transection of the common bile duct as an initial step in multiple organ harvesting. Note that the splenic vein (or alternatively the superior mesenteric vein) is cannulated for eventual delivery of preservation fluid. 
lumbar arteries (Fib. 6B). Then, with a perfect internal view of the renal arterial branches passing laterally, the anterior wall of the aorta is incised longitudinally from the inside (Fig. 6B). If a perfusion is planned, aortic flaps are fashioned during separation and used for closure so that cannulas need not be placed directly into the renal arteries. If the left renal vein was detached from the vena cava as described earlier, it is perfectly safe to divide quickly the remaining structures connecting the right and left kidneys.

After the kidneys or other organs, or both, have been cooled and excised, segments of the iliac arteries and veins are routinely removed (Fig. 7) and placed in a cold tissue culture solution for refrigeration. The thoracic aorta and pulmonary artery may also be taken. Such grafts can be lifesaving in the event of unexpected technical problems in the recipient (7).

\section{TOTAL HEPATECTOMY}

Removal of the liver requires only minor modifications of the foregoing basic technique. As soon as the midline incision is made, the liver is inspected to be sure that its color and texture are normal. Anomalies are looked for, of which arteries to the left lobe from the left gastric artery or to the right lobe from the superior mesenteric artery are the most frequent. Ways of dealing with such anomalies have been described elsewhere $(8,9)$ and will not be considered herein.

If the anatomy is normal, the splenic and left gastric arteries are dissected, ligated and divided (Fig. 8), and the celiac axis is dissected as far back toward the aorta as is convenient. The aorta is encircled at one of the locations above the celiac axis as was described in the section on graft nephrectomy (Figs. 3 and 8).

Turning more distally, the gastroduodenal artery and, when present, the right gastric artery are ligated and divided. Beneath the gastroduodenal artery is found the portal vein (Fig. 8). The common bile duct is mobilized to as low a level as possible and transected (Fig. 8). At the same time, the gallbladder is incised and bile is washed out in order to prevent autolysis of the mucosa of the biliary tract. The portal vein is cleaned inferiorly to the junction of the splenic vein and the superior mesenteric vein. The splenic vein is ligated, and through its central end, a cannula is placed through which a slow infusion of lactated Ringer's solution at 5 degrees $C$. is begun (Fig. 8). The superior mesenteric vein is encircled. If necessary for exposure, the neck of the pancreas should be divided.
Next, the distal part of the abdominal aorta and inferior vena cava are freed and ligated as described in the section on graft nephrectomy (Figs. 2 and 4), but the superior mesenteric artery is not yet tied. Aortic and vena caval cannulas are placed after systemic heparinization. The infusion rate of the cold lactated Ringer's solution through the splenic vein is increased. In adults, the liver can be felt to cool after 1 or 2 liters of infusion at the same time as the body temperature falls. In children, smaller volumes of lactated Ringer's solution are needed for an obvious effect. At this time, the previously encircled superior mesenteric artery (Fig. 8) is tied, followed immediately by ligature of the superior mesenteric vein (Fig. 9). Over infusion is prevented by intermittent bleeding off through the inferior vena cava cannula. The circulation of the donor is still intact and the progressively cooling liver still has an hepatic arterial supply. This has been termed the "pre-cooling" phase (9).

Procurement is terminated as with graft nephrectomy by cross clamping the aorta near the diaphragm at one of the sites of its previous encirclement (Fig. 9). Cold solution through the aortic cannula is immediately infused as with graft nephrectomy, and blood from the inferior vena cava is drained onto the floor bag. The right atrium is also opened as an extra precaution against overdistention of the liver. Anesthesiologic support is stopped. Portal infusion with lactated Ringer's solution is discontinued shortly after aortic cross clamping. After 1 to 2 liters of lactated Ringer's or Collins solution have passed in through the aortic cannula, the kidneys as well as the liver are bloodless and cold. The celiac axis is detached from the aorta with an aortic patch or taken in continuity with the full aortic circumference. If further renal cooling is desired, the donor aorta can be clamped just below the celiac axis.

Until this time, dissection of the suprahepatic vena cava has been avoided. Now, the suprahepatic vena cava is dissected free along with the surrounding cuff of diaphragm. The liver is peeled inferiorly cutting posterior attachments, including the right adrenal veins (Figs. 8 and 9). No unusual effort is made to tie individual tributaries to the vena cava at any level since this can be done later at leisure in an ice basin in the recipient operating room after the specimen has been returned to the parent hospital. Since the celiac axis has already been cut free in continuity with a circumferential piece of aorta or an aortic patch, the liver is ready for removal.

The freed liver is taken to a back table, given a 


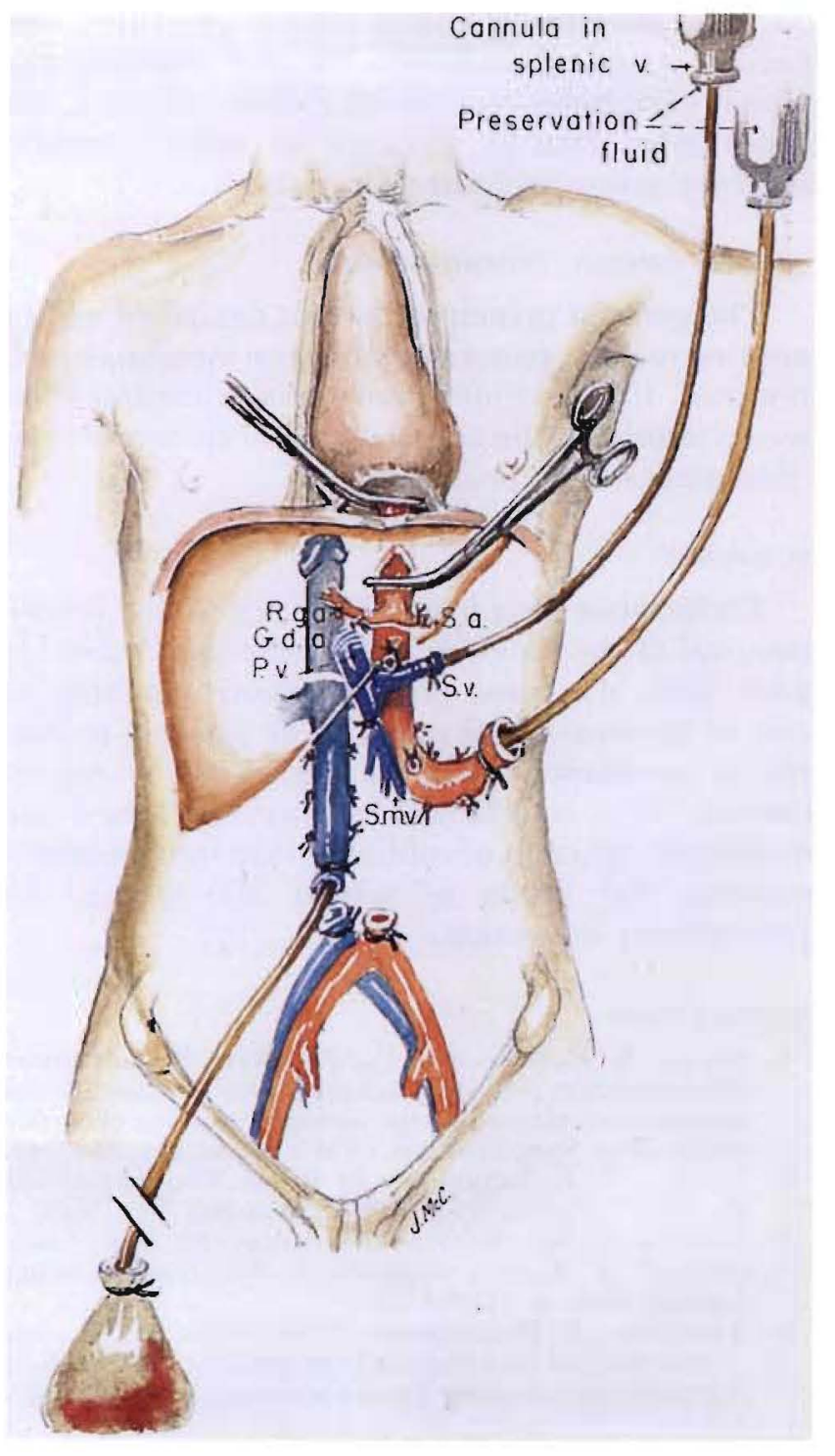

FIG. 9

final flush in adults with 500 or 600 milliliters of cold Collins type solution and placed in a bag which is filled with the same kind of solution. The bag is sealed and covered with crushed ice in a picnic cooler. The ten or 15 minutes required to remove the liver is not harmful to the kidneys which are not subjected to any warm ischemia whatsoever. With the liver out, removal of the kidneys en bloc is carried out as described in the section on graft nephrectomy. The excision is greatly facilitated by the absence of the liver. Vascular grafts are removed as described previously.

\section{REMOVAL OF THE HEART}

With kidneys. The simple preparatory steps described previously are completed. The cardiac team now assumes command. The principle that

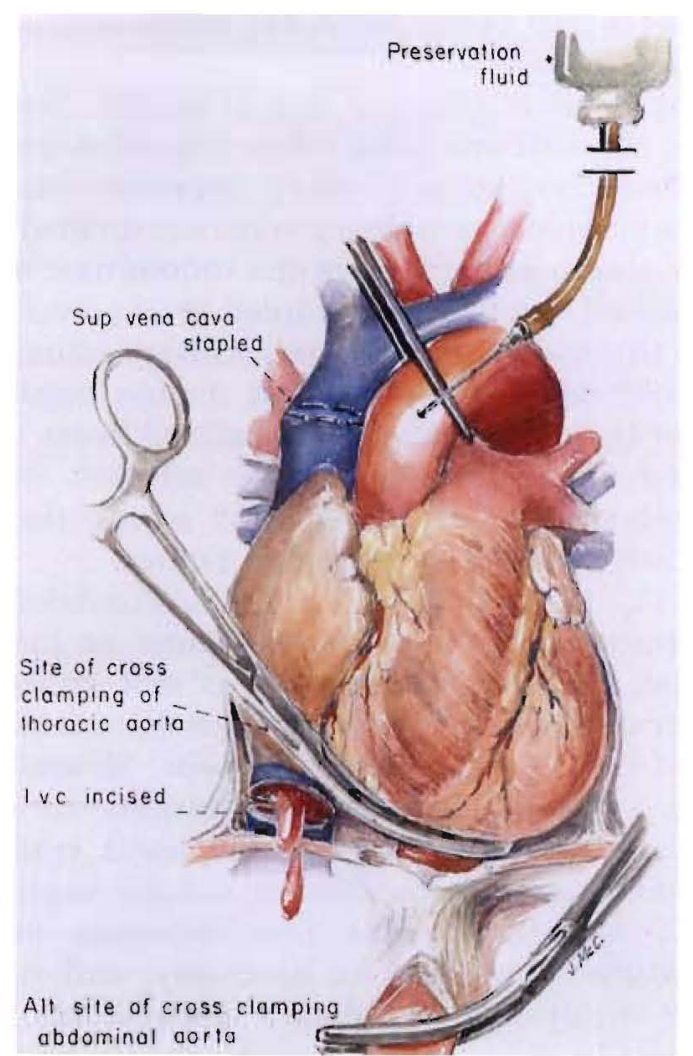

Fig. 10

FIG. 9. In situ infusion technique used when the kidneys and liver are removed from the same donor. R.g.a., Right gastric artery; G.d.a., gastroduodenal artery; S.a., splenic artery; S.u., splenic vein; P.u., portal vein, and S.m.v., Superior mesenteric vein.

FIG. 10. Final steps if the heart is to be removed in combination with nephrectomies or hepatectorny, or both. See text for details.

is followed is to disconnect the thoracic and abdominal aortic circulation by aortic cross clamping at one of the encirclement levels just above or below the diaphragm (Fig. 10) at the precise moment of cessation of heart beat, with immediate graft cooling of both abdominal and thoracic viscera. The preparation for this technical step is the aortic dissection previously described in the section on graft nephrectomy (Fig. 3).

Before this final step is taken, the pericardium is incised, and the aortic root is separated from the main pulmonary artery. The superior vena cava is stapled 2 centimeters proximal to its junction with the right atrium, the inferior vena cava is clamped or incised (Fig. 10) and the beating heart is allowed to empty. At the same time as the aorta is cross clamped at the diaphragm, the thoracic aortic arch is clamped at the origin of the innominate artery. Chilled electrolyte solution is infused into both the aortic root (Fig. 10) and into 
the distal part of the aorta and renal arterial bed (Fig. 4).

The heart is removed first (Fig. 10). Sequentially, the inferior vena cava, left-sided pulmonary veins, pulmonary artery, superior vena cava and right-sided pulmonary veins are divided. The aortic clamp at the level of the innominate artery is removed and the aorta stapled at this level prior to its transection in order to facilitate infusion of the cold cardioplegic solution during implantation of the donor heart. The excised heart is immersed in chilled electrolyte solution during transplantation. After the heart is out, the kidneys are removed as described earlier.

With liver and kidney. The thoracoabdominal disconnection principle is the same as just described. While the circulation is still intact, the intraportal fusion of cold lactated Ringer's solution is begun into the splenic vein. Should ventricular fibrillation occur as a consequence of infusing the chilled solution, the aorta could be clamped, cardioplegia infused and the heart harvested any time. This foreshortening of the procedure should not be necessary, and with a single exception, the heart always has been removed in our experience under the optimal conditions of liver and kidney cooling. The incidental cooling of the heart by cold blood and fluid coming out of the liver may contribute to smooth cardiac cooling. Care is taken not to remove much of the intrapericardial inferior vena cava since it is not important for the cardiac graft but may be needed by the surgeons performing hepatic operations. After the heart is excised, the liver and kidneys are removed in that order.

\section{EXPENDABILITY FACTORS AND GRAFT QUALITY}

The way in which the organs are removed with multiple graft procurement defines an explicit priority list of heart, liver and kidneys in that order. The heart must function immediately and the liver within a few hours, whereas immediate function of renal grafts is not a prerequisite for recipient survival.

Nevertheless, the rate of acute tubular necrosis requiring hemodialysis in patients in whom kidneys were obtained at multiple organ harvests has been only one-fifth of the lowest incidence reported after the harvest of kidneys alone (10-12). The exceedingly low rate could reflect the acceptance of only very good donors for hearts and livers or the fact that there is a high intensity of skilled surgical and anesthesiologic input in such instances. However, the most important factor probably is the systematic use of a superior meth- od of nephrectomy which totally precludes any period of warm ischemia for the kidneys and which eliminates the manipulation that can unknowingly damage kidneys if renal vascular skeletonization techniques are used.

\section{OTHER ORGAN COMBINATIONS}

The general principles herein described can be applied to graft pancreatectomy or intestinal graft removal. If the whole pancreas is transplanted as we recommend, the combination of liver and pancreas removal is incompatible.

\section{SUMMARY}

Techniques have been developed which permit removal of the kidneys, liver, heart and other organs from the same donor without jeopardy to any of the individual grafts. The guiding principle is avoidance with all organs of warm ischemia. This is achieved by carefully timed and controlled infusion of cold solutions into anatomic regions, the limits of which are defined by preliminary dissection.

\section{REFERENCES}

1. Starzl, T. E., Kaupp, H. A., Brock, D. R., and others. Reconstructive problems in canine liver transplantation with special reference to the postoperative role of hepatic venous flow. Surg. Gynecol. Obstet., 1960, 111:733-743.

2. Starzl, T. E. Experience in Renal Transplantation. Pp. 68-71. Philadelphia: W. B. Saunders Co., 1964.

3. Collins, G. M., Bravo-Shugarman, M., and TeraSAKI, P. I. Kidney preservation for transportation. Lancet, 1969, 2: 1219-1222.

4. Benichou, J., Halgrimson, C. G., Weil. III, R., and others. Canine and human liver preservation for 6-18 hours by cold infusion. Transplantation, 1977, 24: 407411.

5. Ackerman, J. R., and Snell, M. E. Cadaveric renal transplantation. Br. J. Urol., 1968, 40: 515-521.

6. Merkel, F. K., Jonasson, O., and Bergan, J. J. Procurement of cadaver donor organs; evisceration technique. Transplant. Proc., 1972, 4: 585-589.

7. Starzl, T. E., Halgrimson, C. G., Koep, L. J., and others. Vascular homografts from cadaveric organ donors. Surg. Gynecol. Obstet., 1979, 149: 737.

8. Starzl, T. E. Experience in Hepatic Transplantation. With the assistance of C. W. Putnam. Philadelphia: W. B. Saunders Co., 1969.

9. Shaw, B. W., Jr., Iwatsuki, S., and Starzl, T. E. Alternative methods of hepatic graft arterialization. Surg. Gynecol. Obstet, in press.

10. Shaw, B. W., JR., Hakala, T., Rosenthai., J. T., and others. Combination donor hepatectomy and nephreclomy and early functional results of allografts. Surg. Gynecol. Obstet., 1982, 155: 321-325.

11. Shaw, B. W., Jr., Rosenthal, J. T., Griffithi, B. P., and others. Techniques for combined procurement of hearts and kidneys with satisfaclory early funciton of renal allografts. Surg. Gynecol. Obstet., 1983, 157: 261264.

12. Shaw, B. W., Jr., Rosenthal, J. T., Griffitif, B. P., and others. Three system organ procurement. Transplant. Proc., 1984, vol. 16. 